[CONTRIBUTIONS FROM THE CHEMICAL LABORATORY OF THE UNIVERSITY OF CINCINNATI.]

\title{
LIII.-ON THE EXISTENCE OF ORTHOSILICIC ACID.
}

By T. H. NORTON AND D. M. ROTh.

Received August 27, 1897.

NUMBER of attempts have been made to ascertain whether
silica combined with water to form a sharply-defined meta-
or orthosilicic acid, and hitherto the results have been very nearly
as fruitless as those undertaken to isolate the corresponding car-
bonic acids. Nearly all experiments in this field have been in
the direction of determining the water remaining in combination
with silica after the precipitated hydrate has been kept in an
air-bath at IOo ${ }^{\circ}$, or in a desiccator over dehydrating substances.
Doveri, the pioneer in this subject, found I 7 to 7.8 per cent.
water in the air-dried powder-source not given. Maschke, ${ }^{2}$
using the hydrate precipitated from alkaline silicates by hydro-
chloric acid, found from I I. 6 to I 4.4 per cent. Fuchs, ${ }^{3}$ Merz ${ }^{4}$
and Gottlieb analyzed the hydrate obtained by the action of water on silicon tetrafluoride. Fuchs found 9.3 per cent. water in a sample dried thirty days over sulphuric acid, and 6.75 per cent. in a sample dried eighteen days at $100^{\circ}$. Merz found for the same 8.66 per cent. and 6.3 I per cent. respectively. Gottlieb, whose experiments were made with the greatest care, found 6. I 3 per cent. Water after desiccation over sulphuric acid for $\mathrm{I} 3 \mathrm{I}$ days and 4.47 per cent. water after desiccation at $100^{\circ}$. His results point to the formulas $\mathrm{H}_{4} \mathrm{Si}_{3} \mathrm{O}_{20}$ and $\mathrm{H}_{4} \mathrm{Si}_{29} \mathrm{O}_{28}$. He concludes, "from these few determinations we may say with certainty that the compound known as orthosilicic acid, having the formula $\mathrm{H}_{4} \mathrm{SiO}_{4}$ does not exist any more than the so-called metasilicic acid $\mathrm{H}_{2} \mathrm{SiO}_{3}$, as the former requires 37.5 per cent., and the latter 23.07 per cent. water, while even an air-dried silicic acid does not contain the latter amount." Rammelsberg" says "Gottlieb found 6.13 per cent. water in the acid dried over sulphuric acid, and 4.50 per cent. water in that dried at $100^{\circ}-140^{\circ}$. I found in the first case, without any special precautions, 4.50 per

\footnotetext{
1 Ann.chim.phys., [3], 21, 40.

2 Pogg. Ann., 556, 9o.

3 Ann.chem. (Liebig), 82, 577 .

4 f. prakt. chem., 99, 177.

$5 \mathrm{~J}$.prakt. chem. $[2], 6,185$.

6 Ber. d.chem. Geir, 5, 1007.
} 
cent., in the second 4.00 to 5.00 per cent. water. We accordingly have to deal with hydrates $n \mathrm{SiO}_{2}+\mathrm{H}_{2} \mathrm{O}$, where $n$ lies between four and eight. Air-dried silicic acid, as one may be easily convinced, contains no certain amount of water. In the dry powder is found from thirty-six to thirteen per cent. water. The former amount would indicate the formula $\mathrm{SiO}_{2}+2 \mathrm{Aq}$, the latter $2 \mathrm{SiO}_{3}+\mathrm{Aq}$. Between these, the figures 23 and 16 per cent. are the most frequent, that is, hydrates $\mathrm{SiO}_{2}+\mathrm{Aq}$ and ${ }_{3} \mathrm{SiO}_{2}+\mathrm{Aq}$." Other results, equally varying, will be found in the work of Lippert, of Fremy, who used silicon sulphide, of Ebelmen, ${ }^{2}$ who used ethyl silicate, Graham, ${ }^{3}$ who employed dialysis, Langlois, ${ }^{4}$ who used silicon chloride, Fullik, ${ }^{5}$ Carnelly and Walker, ${ }^{6}$ and Van Bemmelen. ${ }^{7}$ The latter made an especially exhaustive study of the hydrates obtained from different sources and dried in air saturated with moisture, claiming that as many as four molecules of water would be held in combination under these circumstances, the powder still being dry to the touch.

In order to decide the question, we devised the following method of isolating the hydrate, which led conclusively to the establishment of the formula $\mathrm{H}_{4} \mathrm{SiO}_{4}$ as representing the composition of silicic acid when freshly precipitated, and rapidly freed from adherent water. This was accomplished briefly by washing the moist hydrate with ether or benzene, and removing the latter by pressure between sheets of bibulous paper.

In detail, the following procedure led to the best results : Silicic acid was precipitated by passing silicon tetrafluoride into water. For obvious reasons this is the simplest method of obtaining the compound free from non-volatile foreign matter. The gelatinous mass was thrown upon a cloth filter, washed with water, and allowed to drain a short time. The water was then removed by successive washings with absolute ether or benzene. Usually I 50 to $200 \mathrm{cc}$. were used in an operation. The washed acid was next wrapped in fine cloth, placed between several thicknesses of heavy filter paper and exposed to strong pressure

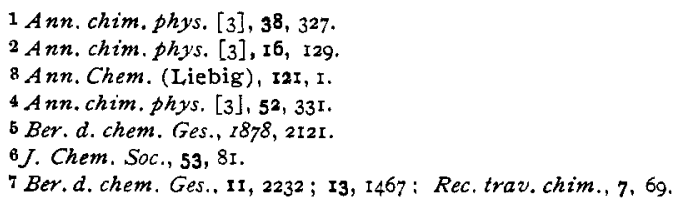


in a vise. After several changes of the paper, and absorption had apparently ceased, the cloth was changed, and the operation repeated. The cake was then removed from the cloth, enclosed in gray filter-paper and pressed again. (Cloth was necessary at first on account of the adherence of paper to the acid. and at the end ordinary coarse gray filter-paper was less torn than the customary white paper.) At this stage the cake was nearly dry. It was broken up, powdered and pressed between paper and porous tiles several times, until all traces of moisture had disappeared. This point was easily detected by the eye. A portion of the perfectly dry mass, which a mounted ordinarily to ten grams at this stage, was transferred to closed watch glasses, and from these weighed off into a platinum crucible. After ignition the amount of silica present and the consequent loss of water, were determined. About forty-five minutes elapsed usually between the beginning of the operation of pressing and the weighing of the sample for analysis. After a little practice it is possible to detect easily the point of disappearance of all moisture, of whatever nature, which is mechanically present. No time is lost at this stage in taking the sample to check the composition, as the loss of chemically combined water begins at once, and its rapidity is dependent only upon prevailing hygroscopic conditions.

The following determinations of orthosilicic acid, as prepared above, were made. Benzene was employed for the washing in Nos. 9, IO, and II; in all other cases absolute ether was used. $\mathrm{H}_{4} \mathrm{SiO}_{4}$ should lose, theoretically, 37.5 per cent. of water.

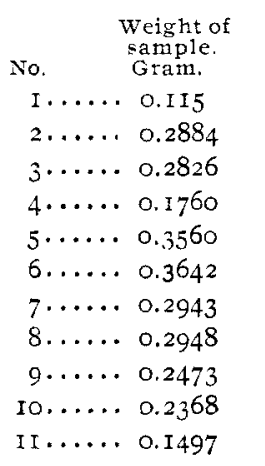

\begin{tabular}{lc}
\multicolumn{2}{c}{ Water lost. } \\
$\begin{array}{l}\text { Weight. } \\
\text { Gram. } \\
0.0428\end{array}$ & 37.30 \\
0.1054 & 36.55 \\
0.1046 & 37.02 \\
0.0660 & 37.50 \\
0.1370 & 38.48 \\
0.1348 & 37.01 \\
0.1098 & 37.31 \\
0.1098 & 37.25 \\
0.0963 & 36.50 \\
0.0873 & 36.60 \\
0.0562 & 37.54
\end{tabular}

Ratio of water
to silica.
I.97:I
I. $92: I$
$I .96: I$
$2.00: I$
$2.08: I$
$1.96: I$
$1.98: I$
$1.98: I$
$I .92: I$
$1.92: I$
$2.00: I$


As was shown by experiment, absolute alcohol could not be used in place of ether or benzene. Its affinity for water is much stronger than that of silicon dioxide, and dehydration began long before the removal of the water mechanically present.

Orthosilicic acid, as obtained above, is an amorphous white powder, perfectly dry to the touch, which may be preserved indefinitely in hermetically closed vessels, but loses its water of hydration steadily on exposure to the air, and especially when in contact with absorbent media.

As examples of this latter property the following analyses were made of (A) samples taken from cakes just dried, and (B) of samples taken from the same cakes, after being pressed for five to ten minutes longer between sheets of filter paper.

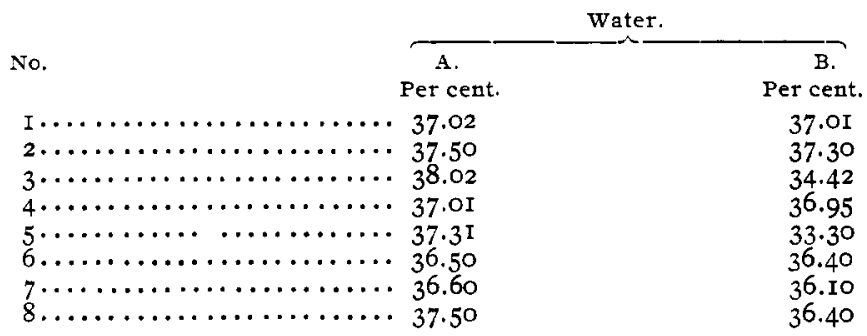

It is probable that in all our experiments a slight loss of water of hydration occurred before the ether or benzene was completely removed by contact with bibulous paper, as the average of the analytical results is slightly below the theoretical figure, 37.5 .

The existence of the orthosilicic acid as a definite body may be therefore considered as established.

[CONTRIBUTIONS FROM THE CHEMICAL, LABORATORY OF THE UNIVERSITY OF CINCINNATI.]

\title{
LIV.-DERIVATIVES OF BENZENESULPHONIC ACID.
}

\author{
BY T. H. NORTON.
}

Received August 27, 1897.

I connection with a general study of the derivatives of benzenesulphonic acid, ${ }^{1}$ carried on in this laboratory, the material for the two following notes was collected.

PREPARATION OF BENZENESULPHONIC BROMIDE.

The only recorded method of preparing the substance is that 1 Norton and Schmidt: $A m$. Chem. $J$, ro, ${ }^{136}$; Norton and Westenhoff : Ibid, so, r29. 